\title{
Active Contour Model Coupling with Higher Order Diffusion for Medical Image Segmentation
}

\author{
Guodong Wang, ${ }^{1}$ Jie Xu, ${ }^{2}$ Qian Dong, ${ }^{3}$ and Zhenkuan Pan ${ }^{1}$ \\ ${ }^{1}$ College of Information Engineering, Qingdao University, Qingdao 266071, China \\ ${ }^{2}$ College of Physics Science, Qingdao University, Qingdao 266071, China \\ ${ }^{3}$ The Affiliated Hospital of Medical College, Qingdao University, Qingdao 266003, China \\ Correspondence should be addressed to Guodong Wang; doctorwgd@gmail.com
}

Received 23 November 2013; Revised 20 January 2014; Accepted 27 January 2014; Published 2 March 2014

Academic Editor: Guowei Wei

Copyright (C) 2014 Guodong Wang et al. This is an open access article distributed under the Creative Commons Attribution License, which permits unrestricted use, distribution, and reproduction in any medium, provided the original work is properly cited.

\begin{abstract}
Active contour models are very popular in image segmentation. Different features such as mean gray and variance are selected for different purpose. But for image with intensity inhomogeneities, there are no features for segmentation using the active contour model. The images with intensity inhomogeneities often occurred in real world especially in medical images. To deal with the difficulties raised in image segmentation with intensity inhomogeneities, a new active contour model with higher-order diffusion method is proposed. With the addition of gradient and Laplace information, the active contour model can converge to the edge of the image even with the intensity inhomogeneities. Because of the introduction of Laplace information, the difference scheme becomes more difficult. To enhance the efficiency of the segmentation, the fast Split Bregman algorithm is designed for the segmentation implementation. The performance of our method is demonstrated through numerical experiments of some medical image segmentations with intensity inhomogeneities.
\end{abstract}

\section{Introduction}

Medical images are popular in real world because they give intuitive expression. With the development of image processing, computer-aided diagnosis are more important with the ever increasing images. Image segmentation is often the first step in computer-aided diagnosis. But medical images are often with intensity inhomogeneities; so it is a hard work to segment in such images as angiogram and MR bladder images.

The active contour model has been increasingly applied to image segmentation in the past decade, because it provides very good frameworks of variational image segmentations. Chan-Vese (CV) model [1] is the most popular active contour model for image segmentation based on the feature of mean gray value of different regions. Chen et al. [2] use variance feature to carry out the segmentation. The variance information can solve the question of different areas with same mean gray value and different variance. The MAP (maximum a posterior) is an efficient region descriptor for the segmentation task. Zhu [3], Paragios and Deriche [4], and Rousson and Deriche [5] approximated the MAP of the given image by a mixture of Gaussians. The features deduced by MAP method are very popular in images with different probability distributions. Sarti et al. [6] deduced using variance information by MAP of Rayleigh distribution.

The above methods are the key development in active contour models. But they are a failure in image segmentation with intensity inhomogeneities, because there are no features that can be deduced for segmentation using the active contour model. The images with intensity inhomogeneities often occurred in real world especially in medical images. Li et al. [7] are the first researchers to deal with this question using region scalable fitting energy. Then, they $[8]$ also extend the method to MRI. Qian et al. [9] use a domain kernel function and an edge indicator function for medical image segmentation. He incorporates the information of different areas and edges for the segmentation.

Pan et al. [10] proposed a segmentation method based on global variables difference. The method is proposed for medical images with complex topological structure, strong contrast, and low noise characteristics. It makes full use of 
the image area information, builds an energy model, and uses variation gradient information to establish a global energy model to get the minimization value. Yao and Cheng [11] use adjustable method for medical image segmentation. They combine active contour model with diffusion filter for multiobject segmentation of the noisy image. A target adaptive scheme is designed for adjusting the model to solving a particular image processing task.

All the methods mentioned above are region-based models aiming to identify each region of interest by using a certain region descriptor to guide the motion of the active contour. For images with intensity inhomogeneity, region descriptor is hard to find. There is much important information such as image gradient and Laplace that are ignored in active contour model. For medical images, intensity inhomogeneity is usually due to technical limitations or artifacts introduced by the object being imaged. The edges are blurred and the gradients are weak. So incorporate edge information alone is not enough, we also incorporate higher order diffusion term into the active contour model aiding segmentation.

In this paper, we propose a new active contour model with gradient and higher-order information of the image. The model can also be deemed as active contour model coupling with high-order diffusion, diffusion, because the introduction of Laplace information and the difference scheme becomes more difficult. To enhance the efficiency of the segmentation, the fast Split Bregman algorithm is designed for the segmentation implementation.

The organization of this paper goes as follows. In Section 2, we will introduce the active contour and highorder diffusion related methods briefly. Then the new active model with high-order diffusion method is proposed in Section 3. Then some numerical examples are shown in Section 4. Section 5 is the concluding remarks.

\section{Higher Diffusion and Active Contour Model}

Generalized Perona-Malik equation for image restoration [12] was the first paper that introduced and implemented high-order anisotropic diffusion partial differential equations (PDEs) for image analysis. There are also many other high-order PDEs that have been already applied to image processing $[13,14]$. They achieved great success in various image processing applications. The models are proposed for staircase effect reduction. Incorporating Laplace term, higher-order diffusion can use more information near the edges. In this paper, we select the total variation with higherorder diffusion because of the simplicity.

The total variation model with higher-order diffusion is depicted as:

$$
\begin{aligned}
E(u)= & \frac{1}{2} \int_{\Omega}(u-f)^{2} d x d y+\lambda_{1} \int_{\Omega}|\nabla u| d x d y \\
& +\lambda_{2} \int_{\Omega}|\Delta u| d x d y
\end{aligned}
$$

where $\Omega$ is the image domain, $u$ is the denoised image, and $f$ is the noisy image.

The active contour model using mean gray value is depicted as:

$$
\begin{aligned}
& \underset{\phi \in[0,1]}{\arg \min }\left\{\int_{\Omega}|\nabla \phi| d x d y\right. \\
& \left.\quad+\int_{\Omega}\left(\alpha_{1}\left|f-u_{1}\right|^{2} \phi+\alpha_{2}\left|f-u_{2}\right|^{2}(1-\phi)\right) d x d y\right\},
\end{aligned}
$$

where $\phi$ is the level set function and $u_{1}$ and $u_{2}$ are the mean gray value of different areas. This contour model is a global convex model proposed by Bresson et al. [15]. The proposed model is a global minimization problem due to convex set $\phi \epsilon$ $[0,1]$. Bresson transformed the original active contour model to a convex minimization problem by relaxing $\phi \in\{0,1\}$ to $\phi \in[0,1]$ and showed that the characteristic function is the global minimizer. This model is based on mean gray value and it has drawbacks with different usages.

For medical images we incorporate the edges and Laplace information into active contour model for dealing with intensity inhomogeneities. The new model has the abilities of both (1) and (2). The new model is depicted in the next section.

\section{Active Contour Model Coupling with Higher-Order Diffusion}

It is hard to segment medical images because the edges are always weak and the images are also noisy. We couple the higher-order diffusion model with active contour model for medical images segmentation. The new model that couples higher-order diffusion with active contour model is

$$
\underset{\phi \in[0,1]}{\arg \min }\left\{\int_{\Omega}|\nabla \phi| d x d y+\int_{\Omega}\left(\begin{array}{c}
\left(\alpha_{1}\left|f-u_{1}\right|^{2}+\beta_{1}\left|\nabla u_{1}\right|+\gamma_{1}\left|\Delta u_{1}\right|\right) \phi \\
+\left(\alpha_{2}\left|f-u_{2}\right|^{2}+\beta_{2}\left|\nabla u_{2}\right|+\gamma_{2}\left|\Delta u_{2}\right|\right)(1-\phi)
\end{array}\right) d x d y\right\},
$$

where $\Omega$ is the image domain, $\alpha_{1}, \beta_{1}, \gamma_{1}, \alpha_{2}, \beta_{2}, \gamma_{2}$ are the positive parameters, $f$ is the original image, $u_{1}$ and $u_{2}$ are the mean values in different image parts, and $\phi$ is a standard level set function.
We use the form of (3) mainly because the image features in different areas are not always the same. This is a natural extension of Chen and Vese [1] and Vese and Chen [16] model for piecewise constant or smooth model. 


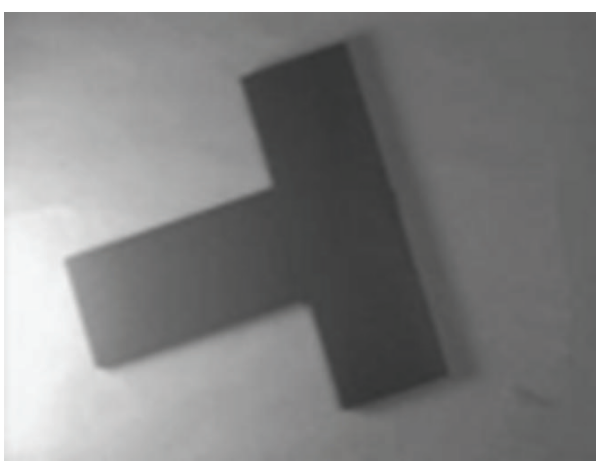

(a)

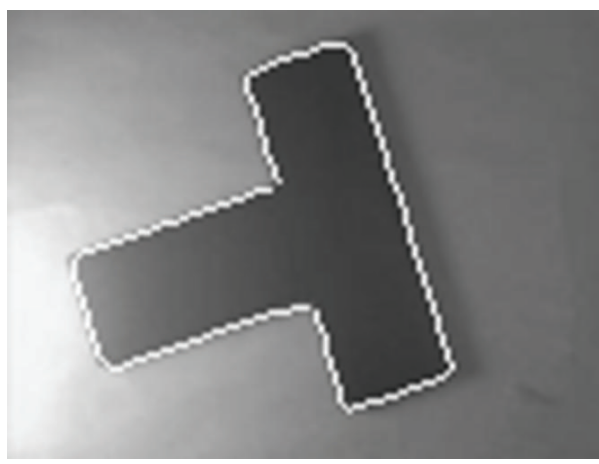

(c)

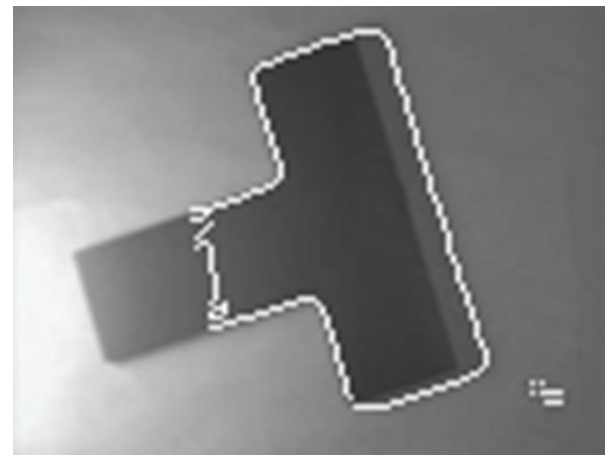

(b)

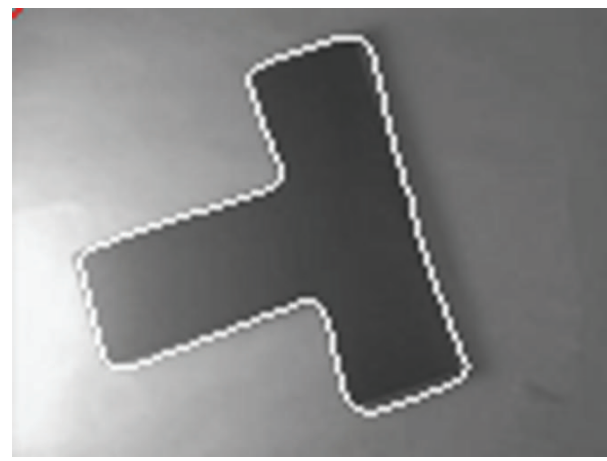

(d)

Figure 1: Image for segmentation. (a) Original image. (b) Result using CV model. (c) Result using mean shift method. (d) Segmentation result using proposed method.

We can get the solution using alternating iteration. Because there are gradient and Laplace information, straightforward solving of (3) by introducing forth-order term of difference scheme is a hard work. The Split Bregman method $[17,18]$ is introduced for the easy implementation of the model.

We introduce the auxiliary variables $\overrightarrow{w_{1}}=\left(w_{11}, w_{12}\right)^{T}, w_{2}$ and Bregman iteration parameters $\vec{b}=\left(b_{1}, b_{2}\right)^{T}$, when the following energy function gets its minimization, $\overrightarrow{w_{1}} \approx$ $\nabla u, w_{2} \approx \Delta u$.

Equation (3) can be divided into the following three subproblems of minimization:

Fix $\phi$ and $u_{2}$, the energy function of (3) became the following form:

$$
\left(u_{1}{ }^{k+1}, \vec{w}_{1}^{k+1}, w_{2}\right)=\underset{\vec{w}_{1}, u_{1}}{\arg \min }\left\{\int_{\Omega}\left(\begin{array}{c}
\beta_{1}\left|\overrightarrow{w_{1}}\right|+\gamma_{1}\left|w_{2}\right|+\alpha_{1}\left|f-u_{1}\right|^{2} \\
+\frac{\mu_{1}}{2}\left(\vec{w}_{1}-\nabla u_{1}-\vec{b}_{1}^{k}\right)^{2}+\frac{\theta_{1}}{2}\left(w_{2}-\nabla \cdot \overrightarrow{w_{1}}\right)^{2}
\end{array}\right) \phi d x d y\right\},
$$

where $\vec{w}_{1}^{k+1}=\nabla u_{1}^{k+1}, \vec{b}_{1}^{k+1}=\vec{b}_{1}^{k}+\nabla u_{1}^{k+1}-\vec{w}_{1}^{k+1}, w_{2}=$ $\nabla \cdot \overrightarrow{w_{1}}, \mu_{1}$, and $\theta_{1}$ are the positive parameters.

The energy function of $u_{1}$ is

$$
E\left(u_{1}\right)=\int_{\Omega}\left(\alpha_{1}\left|f-u_{1}\right|^{2}+\frac{\mu_{1}}{2}\left(\overrightarrow{w_{1}}-\nabla u_{1}-\vec{b}_{1}^{k}\right)^{2}\right) \phi d x d y
$$

The Eular-Lagrange equation is

$$
2 \alpha_{1}\left(u_{1}-f\right) \phi-\mu_{1} \nabla \cdot\left(\phi\left(\nabla u_{1}^{k+1}+{\overrightarrow{b_{1}}}^{k}-{\overrightarrow{w_{1}}}^{k}\right)\right)=0 \text {. }
$$

The discretion of $u_{1}$ is

$$
\begin{aligned}
u_{1}= & \frac{1}{\left(2 \alpha_{1}+4 \mu_{1}\right)} \\
& \times\left(2 \alpha_{1} f+\mu_{1}\left(u_{1, i, j+1}^{k}+u_{1, i, j-1}^{k}\right.\right. \\
& +u_{1, i+1, j}^{k}+u_{1, i-1, j}^{k} \\
& \left.\left.+\nabla \cdot\left(\vec{b}_{1}^{k}-\vec{w}_{1}^{k}\right)\right)\right) .
\end{aligned}
$$




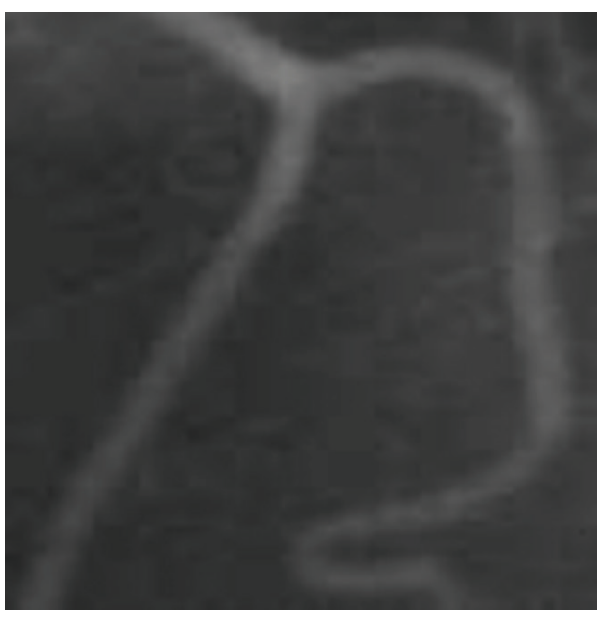

(a)

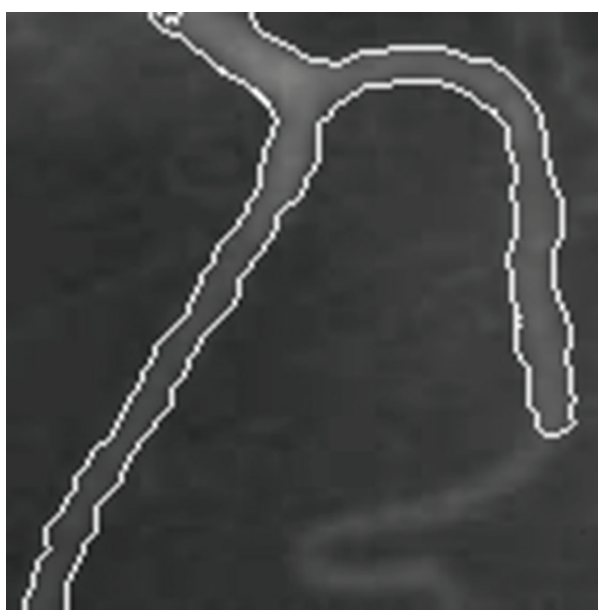

(c)

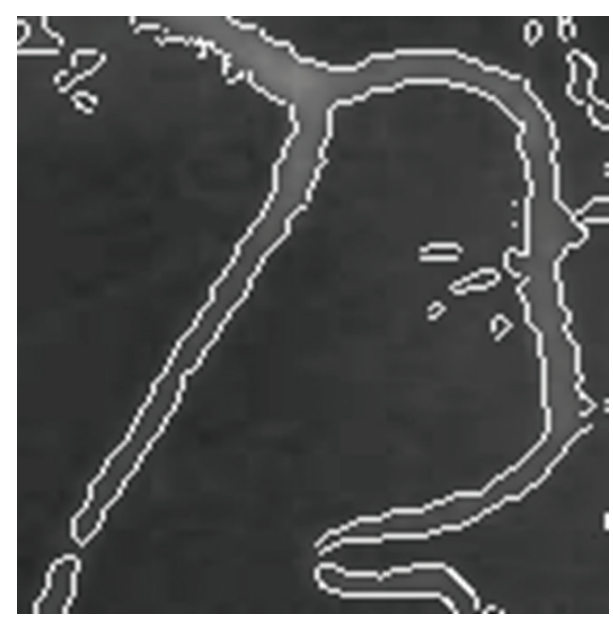

(b)

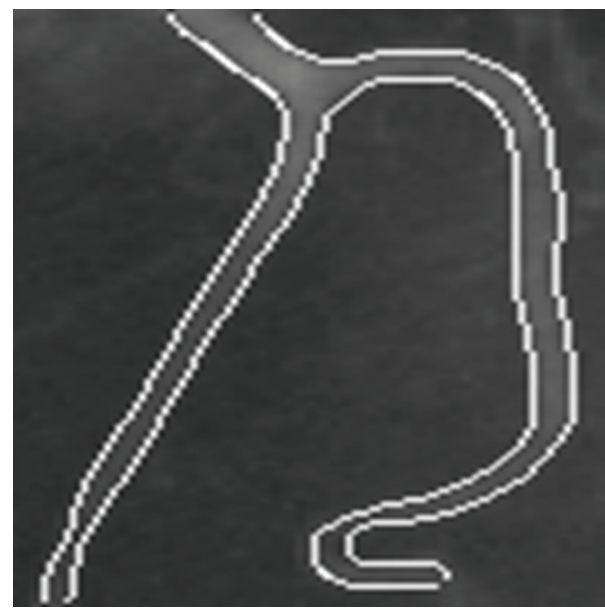

(d)

FIGURE 2: Image for segmentation. (a) Original image. (b) Result using CV model. (c) Result using mean shift method. (d) Segmentation result using proposed method.

The energy function of $\overrightarrow{w_{1}}$ is

$$
\begin{array}{r}
E\left(\overrightarrow{w_{1}}\right)=\int_{\Omega}\left(\beta_{1}\left|\overrightarrow{w_{1}}\right|+\frac{\mu_{1}}{2}\left(\overrightarrow{w_{1}}-\nabla u_{1}-\vec{b}_{1}^{k}\right)^{2}\right. \\
\left.+\frac{\theta_{1}}{2}\left(w_{2}-\nabla \cdot \overrightarrow{w_{1}}\right)^{2}\right) \phi d x d y .
\end{array}
$$

Using Eular-Lagrange equation, we can get

$$
\begin{aligned}
{\overrightarrow{w_{1}}}^{k+1}= & \nabla u^{k+1}+b^{k+1} \\
& +\frac{\theta_{1}}{\mu_{1}} \nabla\left(\nabla \cdot \overrightarrow{w_{1}}-w_{2}\right)-\frac{\beta_{1}}{\mu_{1}} \frac{\vec{w}_{1}^{k+1}}{\left|\vec{w}_{1}^{k+1}\right|}
\end{aligned}
$$

By wavelet soft threshold

$$
\begin{aligned}
\vec{w}_{1}^{k+1}= & \max \left(\left|\nabla u_{1}^{k+1}+{\overrightarrow{b_{1}}}^{k}+\frac{\theta_{1}}{\mu_{1}} \nabla\left(\nabla \cdot \overrightarrow{w_{1}}-w_{2}\right)\right|-\frac{\beta_{1}}{\mu_{1}}, 0\right) \\
& \times \frac{\nabla u_{1}^{k+1}+\vec{b}_{1}^{k}+\left(\theta_{1} / \mu_{1}\right) \nabla\left(\nabla \cdot \overrightarrow{w_{1}}-w_{2}\right)}{\left|\nabla u_{1}^{k+1}+\vec{b}_{1}^{k}+\left(\theta_{1} / \mu_{1}\right) \nabla\left(\nabla \cdot \overrightarrow{w_{1}}-w_{2}\right)\right|} .
\end{aligned}
$$

The energy function of $w_{2}$ is

$$
E\left(w_{2}\right)=\int_{\Omega}\left(\gamma_{1}\left|w_{2}\right|+\frac{\theta_{1}}{2}\left(w_{2}-\nabla \cdot \overrightarrow{w_{1}}\right)^{2}\right) \phi d x d y
$$

Using Eular-Lagrange equation, we can get

$$
w_{2}^{k+1}=\nabla \cdot w_{1}^{k+1}-\frac{\gamma_{1}}{\theta_{1}} \frac{w_{2}^{k+1}}{\left|w_{2}^{k+1}\right|}
$$




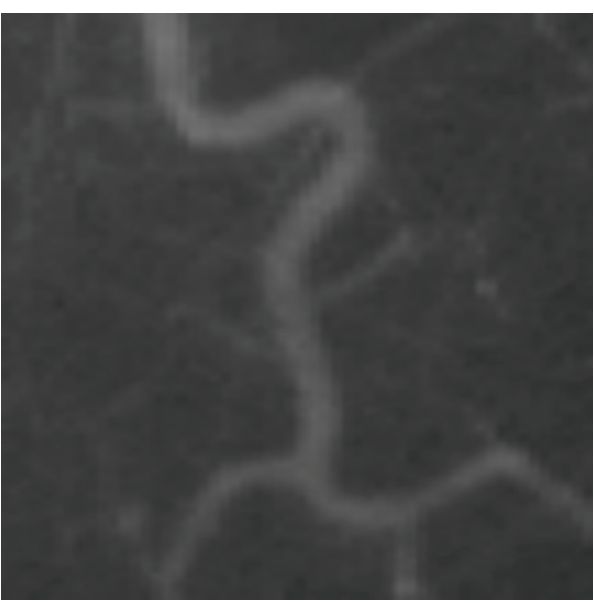

(a)

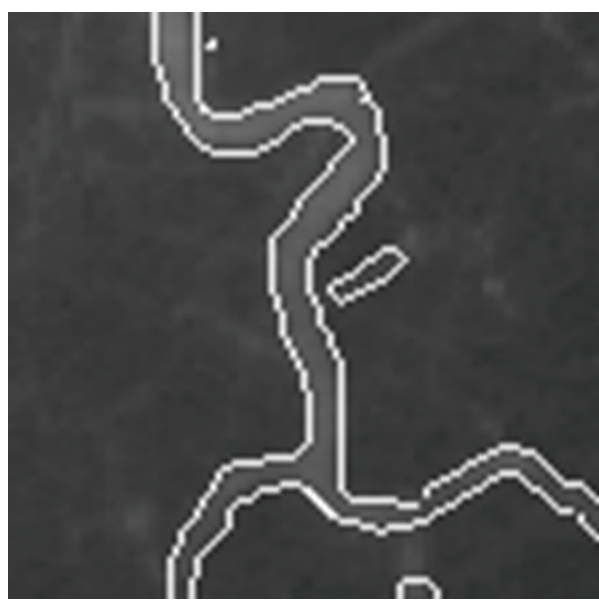

(c)

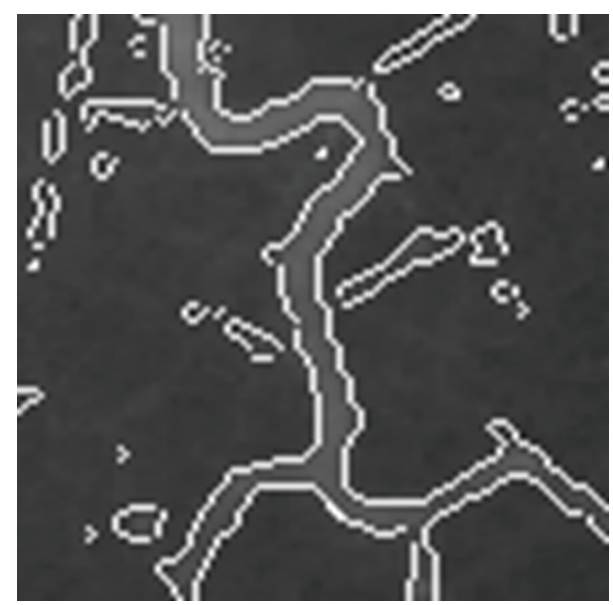

(b)

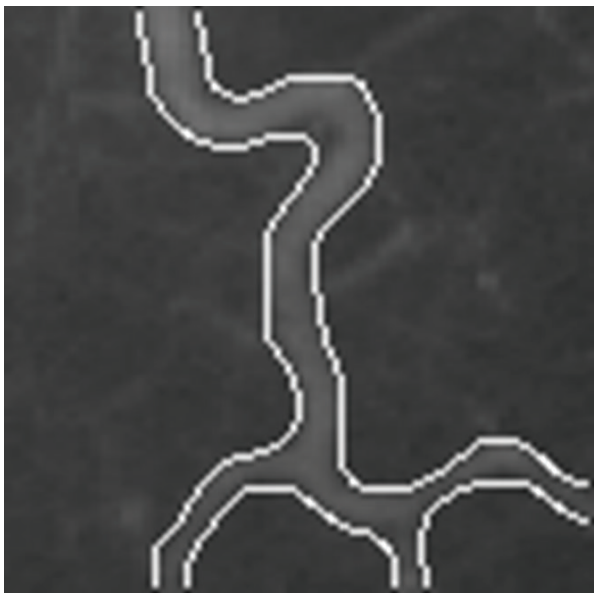

(d)

FIGURE 3: Image for segmentation. (a) Original image. (b) Segmentation result using CV model. (c) Segmentation result using mean shift method. (d) Segmentation result using proposed method.

By wavelet soft threshold,

$$
w_{2}^{k+1}=\max \left(\left|\nabla \cdot w_{1}^{k+1}\right|-\frac{\gamma_{1}}{\theta_{1}}, 0\right) \frac{\nabla w_{2}^{k+1}}{\left|\nabla w_{2}^{k+1}\right|}
$$

For solving $u_{2}$, the procedure is the same as solving $u_{1}$. For convenience, we write

$$
\begin{aligned}
R\left(u_{1}, u_{2}\right)= & \left(\alpha_{1}\left|f-u_{1}\right|^{2}+\beta_{1}\left|\nabla u_{1}\right|+\gamma_{1}\left|\Delta u_{1}\right|\right) \\
& -\left(\alpha_{2}\left|f-u_{2}\right|^{2}+\beta_{2}\left|\nabla u_{2}\right|+\gamma_{2}\left|\Delta u_{2}\right|\right) .
\end{aligned}
$$

The energy function can be rewritten as:

$$
\underset{\phi \in[0,1]}{\arg \min }\left\{E(\phi)=\int_{\Omega}|\nabla \phi| d x d y+\int_{\Omega} R\left(u_{1}, u_{2}\right) \phi d x d y\right\} .
$$

For solving $\phi$, we also use the Split Bregman method by introducing $\vec{v}=\nabla \phi$.
Then the energy function of (14) is transformed as:

$$
\begin{aligned}
& \left(\phi^{k+1}, \vec{v}^{k+1}\right) \\
& =\underset{\vec{d}, \phi \in[0,1]}{\arg \min }\left\{\int_{\Omega}|\vec{v}| d x d y\right. \\
& +\int_{\Omega} R\left(u_{1}, u_{2}\right) \phi d x d y \\
& \left.+\frac{\lambda}{2} \int_{\Omega}\left(\vec{v}-\nabla \phi-\vec{d}^{k}\right)^{2} d x d y\right\},
\end{aligned}
$$

where $\vec{d}^{k+1}=\vec{d}^{k}+\nabla \phi^{k+1}-\vec{v}^{k+1}, \lambda$ is the positive parameter. Fixing $\vec{v}$ for solving $\phi$ :

$$
R\left(u_{1}, u_{2}\right)-\lambda\left(\Delta \phi^{k+1}+\nabla \cdot \vec{d}^{k}-\nabla \cdot \vec{v}^{k}\right)=0
$$




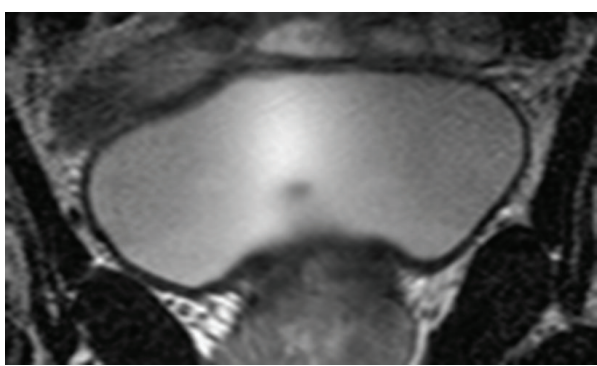

(a)

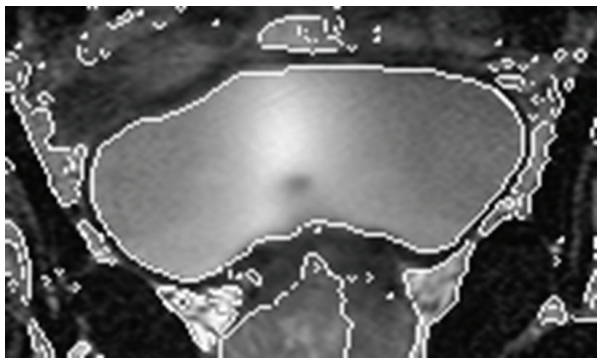

(c)

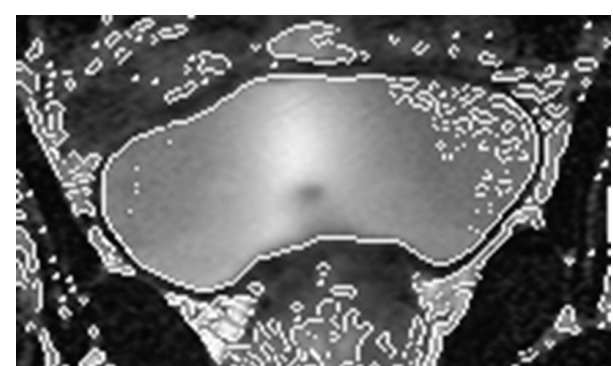

(b)

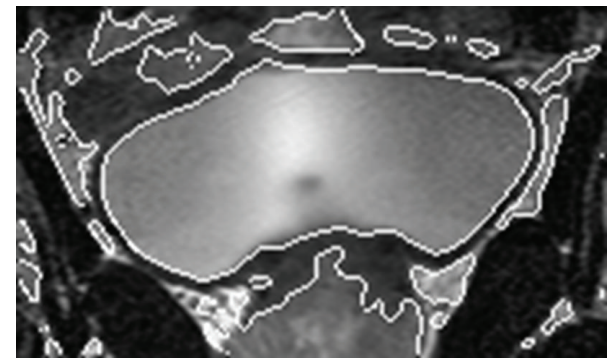

(d)

FIGURE 4: Experiments for an MR image of bladder. (a) Original image. (b) Segmentation result using CV model. (c) Segmentation result using mean shift method. (d) Segmentation result using proposed method.

Using gradient descent method,

$$
\frac{d \phi}{d t}=\lambda\left(\Delta \phi^{k+1}+\nabla \cdot \vec{d}^{k}-\nabla \cdot \vec{v}^{k}\right)-R\left(u_{1}, u_{2}\right)
$$

After discretion,

$$
\begin{aligned}
& \phi^{k+1} \\
& =\left(\frac{1}{1+4 \lambda d t}\right) d t \\
& \times\left(\lambda\left(\phi_{i, j-1}^{k}+\phi_{i, j+1}^{k}+\phi_{i-1, j}^{k}+\phi_{i+1, j}^{k}+\nabla \cdot \vec{d}^{k}-\nabla \cdot \vec{v}^{k}\right)\right. \\
& \left.\quad-R\left(u_{1}, u_{2}\right)\right) .
\end{aligned}
$$

To ensure that $\phi \in[0,1]$, so in every step we use the following function:

$$
\phi^{k+1}=\min \left(\max \left(\phi^{k+1}, 0\right), 1\right) .
$$

Fix $\phi$ for solving $\vec{v}$, we can get

$$
\vec{v}^{k+1}=\max \left(\left|\nabla \phi^{k+1}+\vec{d}^{k}\right|-\frac{1}{\lambda}, 0\right) \frac{\nabla \phi^{k+1}+\vec{d}^{k}}{\left|\nabla \phi^{k+1}+\vec{d}^{k}\right|} .
$$

In the end of calculation, we set

$$
\phi(x)= \begin{cases}0 & \phi(x) \geq \text { th } \\ 1 & \phi(x)<\text { th }\end{cases}
$$

where th is the preset threshold. Thus the foreground and the background can be separated. Due to $\phi \in[0,1]$, our new model is also globally convex. That is to say, the position of $\phi$ need not be initialized.

\section{Numerical Experiments}

To verify the effect of our proposed method, we test our method on a variety of real images with intensity inhomogeneity. We compare our method with CV model [1] and mean shift algorithm [19] because they are highly cited and compared in image segmentation. The CV model which is used in our experiment for comparison is also global convex [15]. For comparison with the mean shift algorithm, we used the software EDISON which is based on a fast implementation of the mean shift algorithm using a speedup scheme described in [19-21].

Figure 1 shows the results for a real image of a T-shaped object with different lighting intensity. Figures 2 and 3 are two experiments on X-ray images of vessels. The images are noisy with intensity inhomogeneity. Figure 4 is a test of MR image of blades with the comparison of $\mathrm{CV}$ model and mean shift method. All of them are selected because they are typical images with intensity inhomogeneity. The CV model and mean shift method cannot get the proper results while our model can get the right results. The shadow of the object is the key element causing wrong segmentation of tradition methods. However, our model can separate the shadow and the object.

The vessel images in Figures 2 and 3 fail to be segmented using traditional methods because of the intensity inhomogeneity. But using our model, the boundary of the object of interest (the bladder) is extracted very well. In these two 
images, parts of the vessel boundaries are quite weak; our method can still segment them well. Satisfactory segmentation results have been obtained for these challenging images. Our method successfully extracts the object boundaries for these two images.

Figure 4 is the segmentation results of an MR image of bladder. The result of mean shift algorithm for the first image is similar to that of our method, showing certain ability of the mean shift algorithm in handling intensity inhomogeneity. However, for the two-vessel image, a small portion of the vessel is missing. For segmenting the MR bladder image, the result is not so perfect for surrounding organs. We notice that the segmentation result of mean shift algorithm is somewhat sensitive to the choice of two major parameters: spatial bandwidth and range bandwidth. We have tweaked these two parameters and other minor parameters for the best segmentation results for these four images.

\section{Conclusion}

In this paper, we present a new active contour model for medical image segmentation. The proposed model incorporates gradient and Laplace information. It can segment images with intensity inhomogeneity and has desirable performance for images with weak object boundaries. Experimental results have demonstrated the advantages of our method over several well-known methods for image segmentation.

\section{Conflict of Interests}

The authors declare that there is no conflict of interests regarding the publication of this paper.

\section{Acknowledgments}

This work was supported by National Natural Science Foundation of China (no. 61305045, no. 61303079, and no. 61170106), National “Twelfth Five-Year” development plan of science and technology (no. 2013BAI01B03), Qingdao science and technology development project (no. 13-1-4-190-jch), and Natural Science Foundation of Shandong Province (no. ZR2010FQ030).

\section{References}

[1] T. F. Chan and L. A. Vese, "Active contours without edges," IEEE Transactions on Image Processing, vol. 10, no. 2, pp. 266-277, 2001.

[2] Y. Chen, H. D. Tagare, S. Thiruvenkadam et al., "Using prior shapes in geometric active contours in a variational framework," International Journal of Computer Vision, vol. 50, no. 3, pp. 315328, 2002.

[3] S. C. Zhu, "Region competition: unifying snakes, region growing, and bayes/mdl for multiband image segmentation," IEEE Transactions on Pattern Analysis and Machine Intelligence, vol. 18, no. 9, pp. 884-900, 1996.

[4] N. Paragios and R. Deriche, "Geodesic active regions: a new framework to deal with frame partition problems in computer vision," Journal of Visual Communication and Image Representation, vol. 13, no. 1-2, pp. 249-268, 2002.

[5] M. Rousson and R. Deriche, "A variational framework for active and adaptative segmentation of vector valued images," in Proceedings of the Workshop on Motion and Video Computing, pp. 56-61, 2002.

[6] A. Sarti, C. Corsi, E. Mazzini, and C. Lamberti, "Maximum likelihood segmentation of ultrasound images with rayleigh distribution," IEEE Transactions on Ultrasonics, Ferroelectrics, and Frequency Control, vol. 52, no. 6, pp. 947-960, 2005.

[7] C. Li, C.-Y. Kao, J. C. Gore, and Z. Ding, "Minimization of region-scalable fitting energy for image segmentation," IEEE Transactions on Image Processing, vol. 17, no. 10, pp. 1940-1949, 2008.

[8] C. Li, R. Huang, Z. Ding, J. C. Gatenby, D. N. Metaxas, and J. C. Gore, "A level set method for image segmentation in the presence of intensity inhomogeneities with application to MRI," IEEE Transactions on Image Processing, vol. 20, no. 7, pp. 20072016, 2011.

[9] X. Qian, J. Wang, S. Guo, and Q. Li, "An active contour model for medical image segmentation with application to brain CT image," Medical Physics, vol. 40, no. 2, pp. 1911-1920, 2013.

[10] Y. Pan, K. Feng, D. Yang, Y. Feng, and Y. Wang, "A medical image segmentation based on global variational level set," in Proceedings of the ICME International Conference on Complex Medical Engineering (CME '13), pp. 429-4432, 2013.

[11] Y. Yao and Y. Cheng, "High effective medical image segmentation with model adjustable method," in Proceedings of the IEEE International Symposium on Circuits and Systems (ISCAS '13), pp. 1512-1515, 2013.

[12] G. W. Wei, "Generalized Perona-Malik equation for image restoration," IEEE Signal Processing Letters, vol. 6, no. 7, pp. 165167, 1999.

[13] G. W. Wei and Y. Q. Jia, "Synchronization-based image edge detection," Europhysics Letters, vol. 59, no. 6, pp. 814-819, 2002.

[14] Y. Wang, G.-W. Wei, and S. Yang, "Partial differential equation transform-Variational formulation and Fourier analysis," International Journal for Numerical Methods in Biomedical Engineering, vol. 27, no. 12, pp. 1996-2020, 2011.

[15] X. Bresson, S. Esedoglu, P. Vandergheynst, J.-P. Thiran, and S. Osher, "Fast global minimization of the active contour/snake model," Journal of Mathematical Imaging and Vision, vol. 28, no. 2, pp. 151-167, 2007.

[16] L. A. Vese and T. F. Chan, "A multiphase level set framework for image segmentation using the Mumford and Shah model," International Journal of Computer Vision, vol. 50, no. 3, pp. 271293, 2002.

[17] S. Simon, "Split bregman algorithm, douglas-rachford splitting and frame shrinkage," in Scale Space and Variational Methods in Computer Vision, vol. 5567 of Lecture Notes in Computer Science, pp. 464-476, Springer, 2009.

[18] J. Yang, W. Yin, Y. Zhang, and Y. Wang, "A fast algorithm for edge-preserving variational multichannel image restoration," SIAM Journal on Imaging Sciences, vol. 2, no. 2, pp. 569-592, 2009.

[19] D. Comaniciu and P. Meer, "Mean shift: a robust approach toward feature space analysis," IEEE Transactions on Pattern Analysis and Machine Intelligence, vol. 24, no. 5, pp. 603-619, 2002.

[20] P. Meer and B. Georgescu, "Edge detection with embedded confidence," IEEE Transactions on Pattern Analysis and Machine Intelligence, vol. 23, no. 12, pp. 1351-1365, 2001. 
[21] C. Christoudias, B. Georgescu, and P. Meer, "Synergism in low level vision," in Proceedings of the 16th International Conference of Pattern Recognition, Quebec City, Canada, August 2001. 

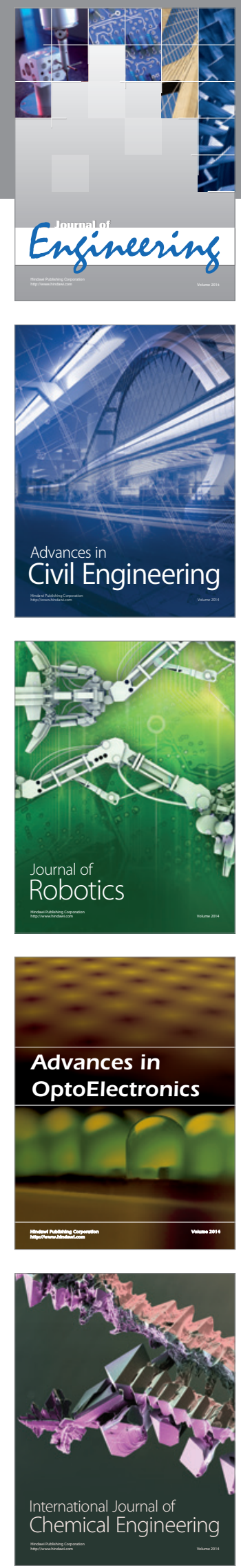

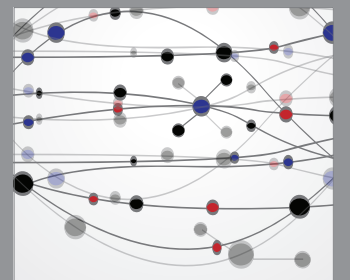

The Scientific World Journal
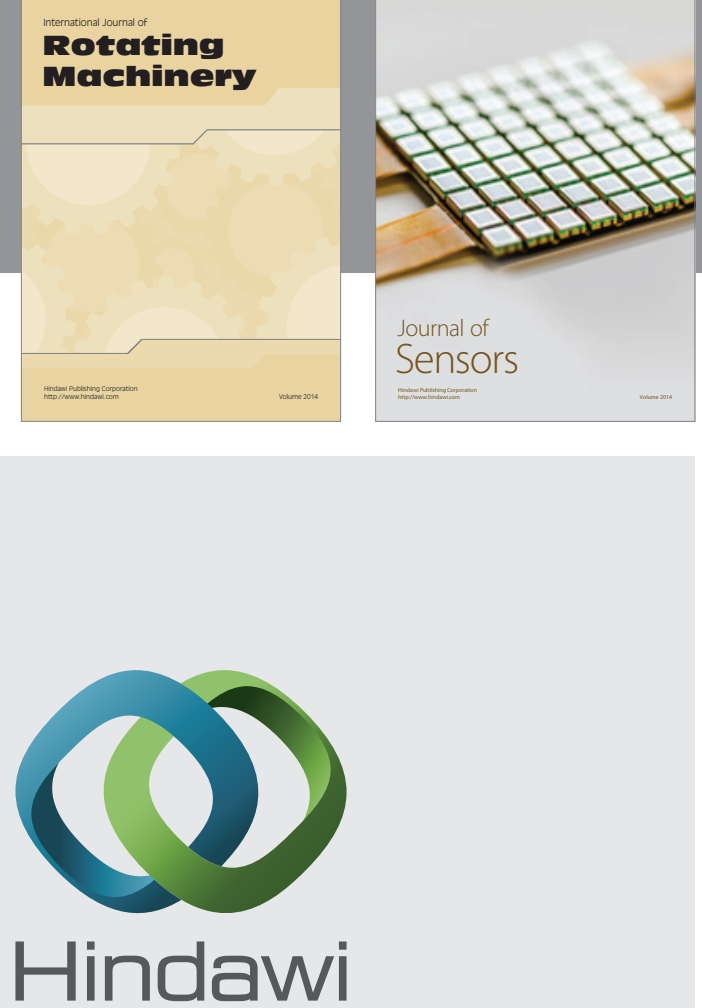

Submit your manuscripts at http://www.hindawi.com
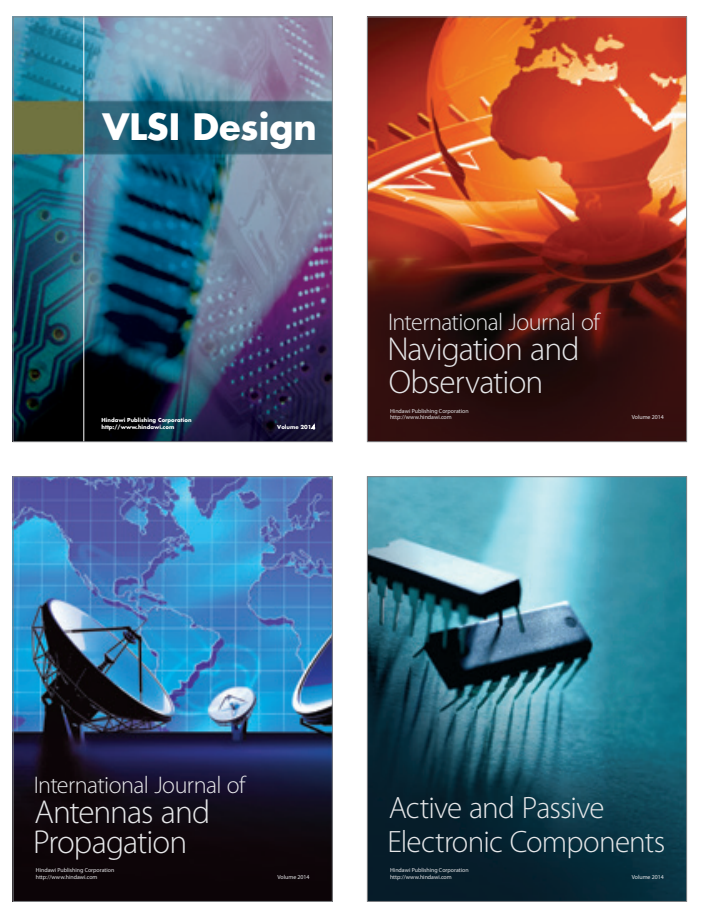
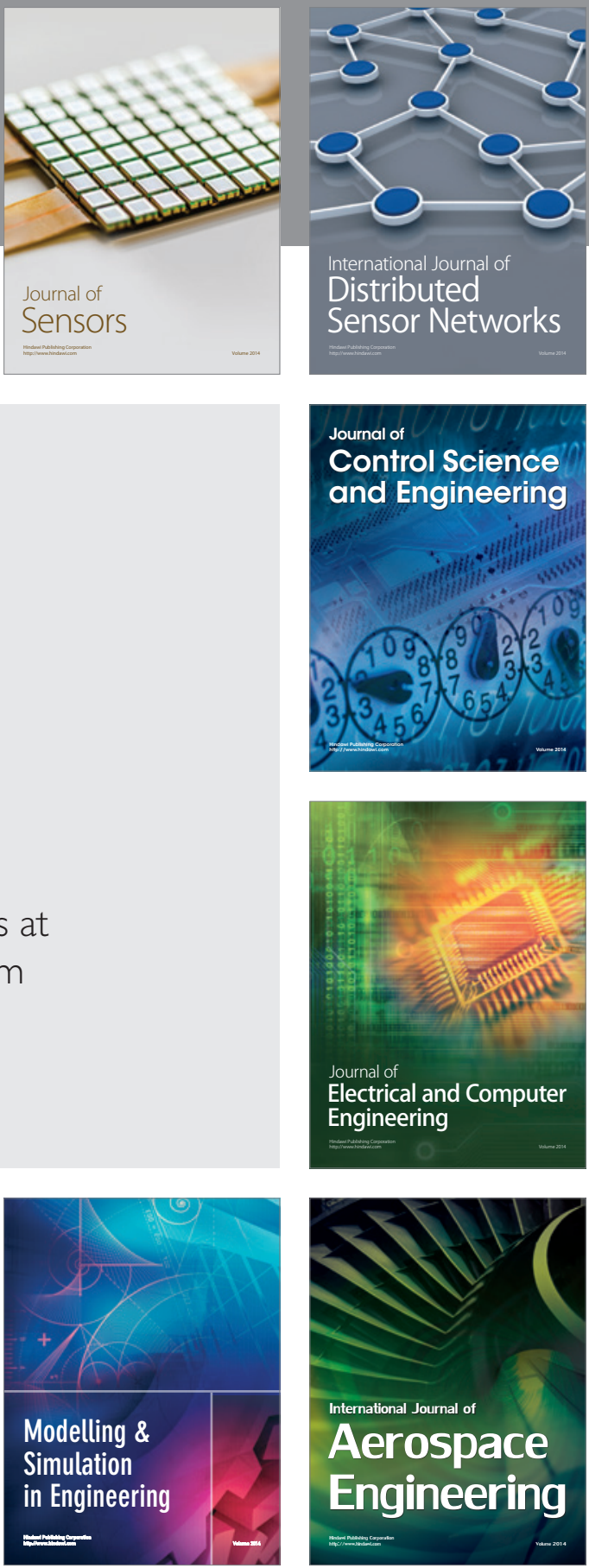

Journal of

Control Science

and Engineering
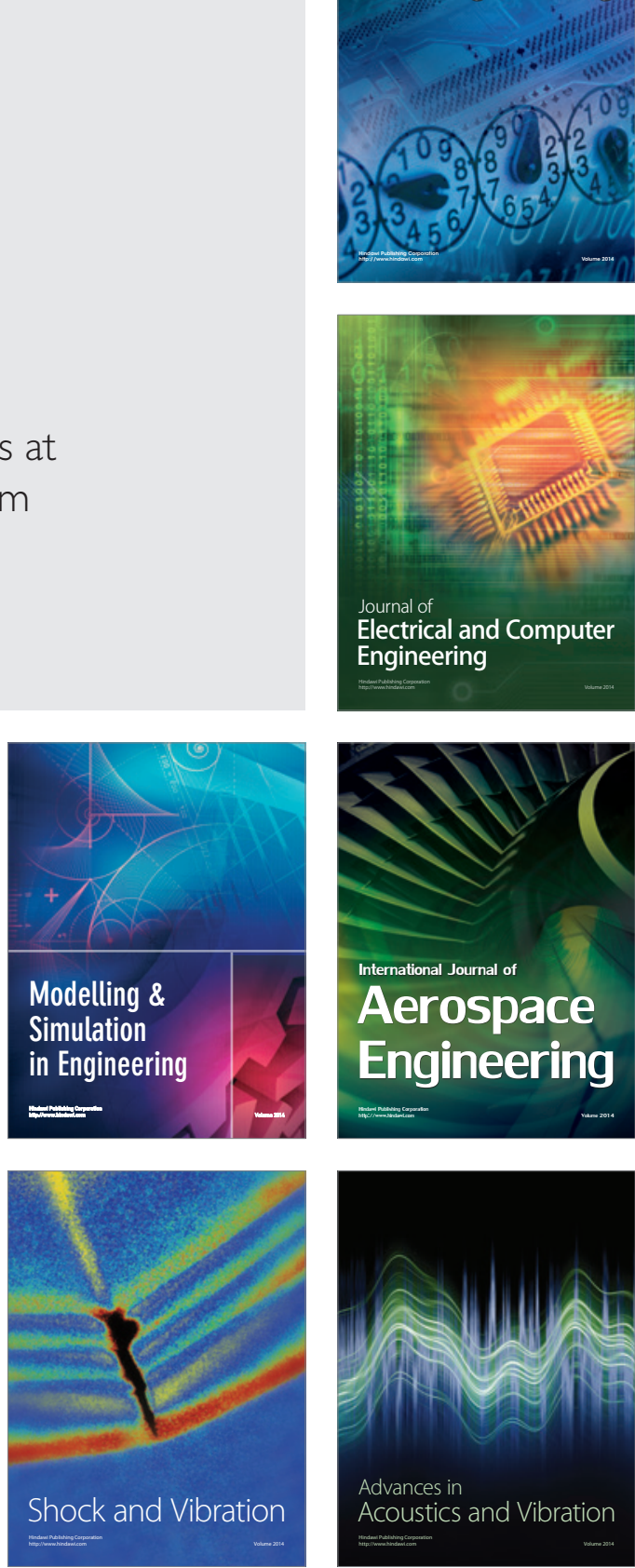